\title{
Evaluating extremely low plasma ascorbate levels and reduction of plasma ascorbate levels by dialysis in Japanese hemodialysis patients
}

\section{Yuta Doshida}

Tokyo-to Kenko Choju Iryo Center

Mitsuyo Itabashi

Tokyo-to Kenko Choju Iryo Center

Takashi Takei

Tokyo-to Kenko Choju Iryo Center

\section{Yuka Takino}

TMIG

Ayami Sato

Tokyo-to Kenko Choju Iryo Center

\section{Tomofumi Yatsu}

Tokyo-to Kenko Choju Iryo Center

Wako Yumura

Tokyo-to Kenko Choju Iryo Center

Naoki Maruyama

Tokyo-to Kenko Choju Iryo Center

Akihito Ishigami ( $\square$ ishigami@tmig.or.jp )

TMIG https://orcid.org/0000-0001-7875-7482

\section{Research article}

Keywords: ascorbate, chronic kidney disease (CKD), hemodialysis, hyperkalemia, oxalate, potassium, scurvy, vitamin C

Posted Date: May 21st, 2020

DOI: https://doi.org/10.21203/rs.3.rs-29513/v1

License: (c) (1) This work is licensed under a Creative Commons Attribution 4.0 International License.

Read Full License 


\section{Evaluating extremely low plasma ascorbate levels and reduction of plasma}

\section{2 ascorbate levels by dialysis in Japanese hemodialysis patients}

3

4 Yuta Doshida 1,2,\#, Mitsuyo Itabashi 1,3,\#, Takashi Takei 1,3,\#, Yuka Takino ${ }^{1}$, Ayami Sato ${ }^{1}$,

5 Tomofumi Yatsu ${ }^{1}$, Wako Yumura ${ }^{1,3,4}$, Naoki Maruyama ${ }^{1,5}$, Akihito Ishigami 1,2,*

6

$7{ }^{1}$ Molecular Regulation of Aging, Tokyo Metropolitan Institute of Gerontology, Tokyo 173-

80015 , Japan

$9 \quad 2$ Department of Biological Sciences, Tokyo Metropolitan University, Tokyo 192-0397, Japan

$10{ }^{3}$ Department of nephrology, Tokyo Metropolitan Geriatric Hospital, Tokyo 173-0015, Japan

$11{ }^{4}$ Department of Nephrology and Endocrinology, Tohoku Medical and Pharmaceutical

12 University Hospital, Miyagi 983-8512, Japan

135 Saitama Central Hospital, Saitama 354-0045, Japan

14

15 \# These authors contributed equally to this work. 
17 * Corresponding author: Akihito Ishigami, Ph.D., Molecular Regulation of Aging, Tokyo

18 Metropolitan Institute of Gerontology (TMIG), 35-2 Sakae-cho, Itabashi-ku, Tokyo 173-0015,

19 Japan. Phone +81-3-3964-3241, E-mail: ishigami@tmig.or.jp

20

21 Abbreviations: ANOVA, analysis of variance; AST, aspartate aminotransferase; CKD,

22 chronic kidney disease; COPD, chronic obstructive pulmonary disease; DHA, dehydroascorbic

23 acid; EDTA, ethylenediaminetetraacetic acid; HbA1c, hemoglobin A1c; LDL, low-density

24 lipoprotein; TIBC, iron-binding capacity; SEM, standard error of the mean; RDA,

25 recommended dietary allowance 


\section{Abstract}

29 Background: Low plasma ascorbate levels in hemodialysis patients have been reported worldwide; hence, many end-stage kidney disease patients are forced to restrict their diets, especially potassium-rich fruits and vegetables, to prevent hyperkalemia. In this study, we aimed to clarify whether plasma ascorbate levels are low in Japanese dialysis patients and whether plasma ascorbate levels fluctuate before and after dialysis. In addition, we aimed to clarify whether there are clinical test items that have a causal relationship with plasma ascorbate levels.

Methods: Plasma ascorbate levels in 27 chronic kidney disease (CKD) stage G3-G5 patients

37 (mean age 84 years) and pre- and post-dialysis plasma ascorbate levels in 19 CKD stage G5D

38 hemodialysis patients (mean age 79 years) were determined using high-performance liquid

39 chromatography and electrochemical detection.

40 Results: Pre-dialysis plasma ascorbate levels in hemodialysis patients $(12.0 \pm 1.4 \mu \mathrm{M})$ were

41 significantly lower (by 56\%) than those in CKD stage G3-G5 patients $(27.1 \pm 2.7 \mu \mathrm{M})$. After dialysis, there was a 40\% reduction in plasma ascorbate levels. Moreover, pre-dialysis ascorbate levels correlated significantly with plasma potassium levels. 
44 Conclusions: The study results indicate that Japanese hemodialysis patients have lower plasma

45 ascorbate levels than CKD stage G3-G5 patients and that these low plasma ascorbate levels in

46 hemodialysis patients were further reduced by hemodialysis. To avoid the development of

47 scurvy in hemodialysis patients, it is necessary to take sufficient ascorbate from supplements

48 or medicines.

49

50 Keywords: ascorbate; chronic kidney disease (CKD); hemodialysis; hyperkalemia; oxalate;

51 potassium; scurvy; vitamin C

52 


\section{Background}

54

55 Vitamin C (L-ascorbic acid) is a water-soluble micronutrient and antioxidant that scavenges

56 reactive oxygen species [1-3]. Under physiological $\mathrm{pH}$ conditions, ascorbic acid most

57 commonly exists in its mono-anion form, ascorbate [4]. In addition to its antioxidant property,

58 ascorbate contributes to numerous well-defined enzymatic reactions involving collagen

59 hydroxylation, carnitine and norepinephrine biosynthesis, tyrosine metabolism, and peptide

60 hormone amidation [5-7]. Many vertebrates have the ability to synthesize ascorbate from

61 glucose de novo in the liver [8]. However, primates, including humans, are unable to synthesize

62 ascorbate since they carry multiple mutations in the Gulo gene encoding L-gulono- $\gamma$-lactone

63 oxidase, the last enzyme in the ascorbate biosynthesis pathway [9]. Therefore, humans must

64 consume ascorbate from dietary sources such as fresh fruits and vegetables to prevent scurvy.

65 Scurvy is a condition that results from insufficient ascorbate in the body. Most scurvy

66 symptoms such as anemia, weakness, and gingival bleeding are often seen in hemodialysis

67 patients [10].

68 In the recent years, the increasing number of patients undergoing dialysis has become a social

69 problem worldwide. Currently, there has been an increase in the mean age of incident dialysis 
among patients aged $\geq 45$ years, especially among those aged $\geq 65$ years [11]. The proportion of

71 patients aged $\geq 65$ years at the end of 2012 was $65.5 \%$ in Japan, indicating the increase in

72 dialysis incidence in the aging population [11].

73 Low plasma ascorbate levels have been observed in some hemodialysis patients for many years

74 globally [12-18]. However, it is unclear why plasma ascorbate levels are low in hemodialysis

75 patients. Hemodialysis patients are forced to have dietary restrictions, such as protein, salt, and

76 potassium restriction. The consumption of fruits and vegetables that contain high amounts of

77 ascorbate is also restricted due to the high potassium content.

78 In this study, we examined whether Japanese hemodialysis patients have low plasma ascorbate

79 levels compared to non-hemodialysis-dependent patients with chronic kidney disease (CKD).

80 In addition, we also examined whether dialysis reduces plasma ascorbate levels. Furthermore,

81 we analyzed whether there are any clinical test items that have a causal relationship with plasma

82 ascorbate levels in Japanese hemodialysis patients.

83 


\section{Methods}

85

86

Ethical consideration

87 This study was conducted according to the principles expressed in the Declaration of Helsinki.

88 This study was approved by the Clinical Research Ethics Committee of the Tokyo Metropolitan

89 Geriatric Medical Center, Tokyo, Japan (permit number: R18-40) and Tokyo Metropolitan

90 Institute of Gerontology, Tokyo, Japan (permit number: H30-25). All patients gave their written

91 informed consent and patient anonymity is preserved.

92

93 Study patients

94 Patients with CKD stage G3-G5 (mean eGFR $\left.23 \mathrm{~mL} / \mathrm{min} / 1.73 \mathrm{~m}^{2}\right)(\mathrm{n}=34)$ and CKD stage G5D

95 hemodialysis $(\mathrm{n}=19)$ who had been regularly visiting an outpatient clinic of Tokyo Metropolitan

96 Geriatric Medical Center from October 2018 through December 2019 were recruited in this

97 study. A diagnosis of CKD was made based on the guidelines of the National Kidney

98 Foundation Kidney Disease Outcomes Quality Initiative [19].

99 
101 Of the 19 patients, 10 were under maintenance hemodialysis and 9 patients were undergoing

102 hemodiafiltration. The procedures were performed three times weekly for $9-12 \mathrm{~h}$ per week at

103 a blood flow rate of $150-200 \mathrm{~mL} / \mathrm{min}$ and dialysis flow rate of $500 \mathrm{~mL} / \mathrm{min}$ using dialyzers

104 with a surface area of $1.1-2.1 \mathrm{~m}^{2}$. The dialysate sodium concentration was $140 \mathrm{mEq} / \mathrm{L}$ and the

105 potassium concentration was $2.0 \mathrm{mEq} / \mathrm{L}$. The mean duration of dialysis therapy was 3.6 years.

106 The mean $\mathrm{Kt} / \mathrm{V}$ was 1.3.

107

108 Collection of blood and urine samples

109 Blood samples for the measurement of clinical test items and ascorbate were obtained at the

110 same time. For the determination of ascorbate levels, blood samples were drawn into a

111 VENOJECT $^{\circledR}$ collection tube (Terumo Corporation, Tokyo, Japan) containing

112 ethylenediaminetetraacetic acid (EDTA)-2Na as an anticoagulant. All of the following

113 procedures were performed within $2 \mathrm{~h}$ after sampling since we confirmed in advance that the

114 values of ascorbate are unstable if it is beyond $2 \mathrm{~h} \mathrm{[20].} \mathrm{Plasma} \mathrm{was} \mathrm{obtained} \mathrm{by} \mathrm{centrifugation}$

115 at $1,700 \mathrm{~g}$ for $10 \mathrm{~min}$. After the plasma was collected, $0.5 \mathrm{~mL}$ of supernatant was immediately

116 mixed with $0.5 \mathrm{~mL}$ of cold $10 \%$ metaphosphoric acid (Wako Pure Chemical Industries, Ltd.,

117 Osaka, Japan) containing $1 \mathrm{mmol} / \mathrm{L}$ of EDTA (Dojindo Laboratories, Kumamoto, Japan) and 
119 the urine samples, $0.5 \mathrm{~mL}$ of urine was immediately mixed with $0.5 \mathrm{~mL}$ of cold $10 \%$ metaphosphoric acid containing $1 \mathrm{mmol} / \mathrm{L}$ of EDTA and centrifuged at 21,000 $\mathrm{g}$ for $15 \mathrm{~min}$ at

\section{Collection of blood from dialysis}

124 To establish the basal plasma ascorbate levels in hemodialysis patients and the effect of

125 hemodialysis, blood samples were drawn (from the arteriovenous fistula or catheter) prior to

126 the start of dialysis (pre-dialysis sample) and immediately after ending the dialyzing period

127 (post-dialysis sample).

129 Determination of ascorbate and dehydroascorbic acid (DHA)

130 Ascorbate and DHA, which is an oxidized form of L-ascorbic acid, levels were measured using

131 high-performance liquid chromatography and electrochemical detection according to the methods described previously [21]. After thawing, the plasma and urine were centrifuged at 
135 After reduction, the reaction mixture was diluted with 5\% metaphosphoric acid containing 0.5

mmol/L EDTA and analyzed for total ascorbate by high-performance liquid chromatography

coupled with electrochemical detection. Separation was achieved on an Atlantis dC18 5- $\mu \mathrm{m}$

column $(4.6 \times 150 \mathrm{~mm})$ combined with an Atlantis $\mathrm{dC} 185-\mu \mathrm{m}$ guard column $(4.6 \times 20 \mathrm{~mm})$

(pH 2.8), $540 \mu \mathrm{M}$ EDTA, and $2 \%$ methanol at a flow rate of $1.3 \mathrm{~mL} / \mathrm{min}$, and electrical signals evaluated the plasma ascorbate level in the samples whose urinary ascorbate was $<0.5 \mathrm{mM}$.

\section{Study items}

148 The following data were collected from the medical records: age, gender, and clinical

149 investigations, i.e., white blood cell, hemoglobin, hematocrit, platelet, total protein, albumin, 
152 (LDL) cholesterol, iron, transferrin and iron-binding capacity (TIBC), ferritin, $\beta 2$ -

153 microglobulin, prealbumin, hemoglobin A1c (HbA1c), and parathyroid hormone.

154

155 Statistical analysis

156 The results and clinical characteristic data are expressed as means \pm standard error of the mean

157 (SEM). The probability of statistical differences between experimental groups was determined

158 by Welch's t-test, paired t-test, and one-way analysis of variance (ANOVA) followed by

159 Tukey-Kramer test. We verified that Pearson correlation coefficient between ascorbate

160 concentration and clinical characteristics data is different from zero. Statistical differences were

161 considered significant at $p<0.05$.

162 


\section{Results}

\section{Clinical characteristics of CKD stage G3-G5 and hemodialysis patients}

166 A total of 34 CKD stage G3-G5 and 19 CKD stage G5D hemodialysis patients were enrolled.

167 Since seven CKD stage G3-G5 patients showed that urine ascorbate level was higher than 0.5

$168 \mathrm{mM}$, suggesting supplemental intake of ascorbate just before collecting blood and urine, they

169 were excluded from analysis. Therefore, the results of 27 CKD stage G3-G5 patients were used

170 for analysis. Clinical characteristics of CKD stage G3-G5 and hemodialysis patients are shown

171 in Table 1 . The levels of the following were beyond the normal range and differed between

172 CKD stage G3-G5 and hemodialysis patients: albumin, blood urea nitrogen, creatinine,

173 phosphorus, and $\beta 2$-microglobulin. Albumin values of both CKD stage G3-G5 and

174 hemodialysis patients were below the normal range and blood urea nitrogen, creatinine, and $\beta 2$ -

175 microglobulin values of both CKD stage G3-G5 and hemodialysis patients were higher than

176 the normal range. Phosphorus values of hemodialysis-only patients were higher than the normal

177 range. 

ascorbate levels in hemodialysis patients

The plasma ascorbate levels in 27 CKD stage G3-G5 and pre- and post-dialysis plasma in CKD stage G3-G5 patients and pre- and post-dialysis plasma ascorbate levels in hemodialysis patients are shown in Figure $1 \mathrm{~b}$ and $\mathrm{c}$.

191 We then analyzed the relationships between clinical characteristics and pre-dialysis plasma

192 ascorbate levels in hemodialysis patients (Fig. 2). In Pearson correlation coefficient, pre-

193 dialysis ascorbate levels correlated significantly with those of plasma potassium levels (positive correlation; Pearson correlation coefficients $(\mathrm{r})=0.6 ; p=0.006$ ) (Table 2). However, no 
195 association was found between plasma ascorbate levels and other clinical characteristics except

196 for plasma potassium levels.

197 


\section{Discussion}

199

200 In this study, we revealed that Japanese hemodialysis patients have low plasma ascorbate levels

201 compared to non-hemodialysis CKD patients and these low plasma ascorbate levels in

202 hemodialysis patients were further reduced by a single hemodialysis treatment session.

203 Moreover, we found that ascorbate levels in hemodialysis patients correlated with those of

204 plasma potassium levels. In general, hemodialysis patients are forced to restrict their diets,

205 especially potassium-rich fruits and vegetables, to prevent hyperkalemia, which is a risk factor

206 for dialysis morbidity and mortality [22]. Most of these fruits and vegetables also contain high

207 amounts of ascorbate. Thereby, plasma ascorbate levels in Japanese hemodialysis patients

208 might be correlated with plasma potassium levels.

209 The recommended dietary allowance (RDA) of vitamin C for a healthy adult is $100 \mathrm{mg}$ per day

210 in Japan and $90 \mathrm{mg}$ and $75 \mathrm{mg}$ per day for men and women, respectively, in the United States

211 to prevent scurvy [23]. The average concentration of ascorbate in the plasma of healthy humans

212 is $40-60 \mu \mathrm{M}[24,25]$. When the plasma ascorbate concentration drops to below $11 \mu \mathrm{M}$, there

213 is a risk of developing scurvy, which is thus conventionally considered deficient [24, 25]. In

214 our previous report regarding chronic obstructive pulmonary disease (COPD) and plasma 
215 ascorbate levels, we reported that plasma ascorbate levels were significantly lower in COPD

216 patients (mean age $72.7 \pm 6.9$ years) than those in healthy elderly people (mean age $68.8 \pm 3.8$

217 years) using the same procedure and method as here [20]. Plasma ascorbate levels in COPD

218 patients and healthy elderly people were $31.2 \pm 2.2 \mu \mathrm{M}$ and $42.3 \pm 2.9 \mu \mathrm{M}$, respectively.

219 Furthermore, the observed plasma levels in non-hemodialysis CKD patients and hemodialysis

220 patients in the present study were lower than those in COPD patients. Plasma ascorbate levels

221 in non-hemodialysis CKD patients and hemodialysis patients were $27.1 \pm 2.7 \mu \mathrm{M}$ and $12.0 \pm$

$2221.4 \mu \mathrm{M}$, respectively. Since there is a risk of developing scurvy when the plasma ascorbate

223 concentration drops to below $11 \mu \mathrm{M}[24,25]$, many Japanese hemodialysis patients are likely

224 develop scurvy (see Fig. 3). Worldwide, many hemodialysis patients have developed scurvy

$225[10,26,27]$.

226 Moreover, we tried to compare the percentages of DHA per total ascorbate in plasma from

227 healthy elderly people, COPD patients, non-hemodialysis CKD patients, and hemodialysis

228 patients, and found that the percentages of DHA in non-hemodialysis CKD patients (33.5\%)

229 and hemodialysis patients (37.4\%) were notably higher percentages than those in COPD

230 patients (12.4\%) and healthy elderly people (10.0\%) (see Fig. 3) [20]. High percentage of DHA 
231 in non-hemodialysis CKD patients and hemodialysis patients may reflect a higher oxidative

232 stress levels in their body.

233 Wang et al. [16] reported that plasma ascorbate concentrations were reduced by a median of

$23433 \%$ following dialysis. Deicher et al. [28] have also reported that hemodialysis causes a 50-

$23575 \%$ decrease in plasma ascorbate levels. In the present study, plasma ascorbate levels reduced

236 to $40 \%$ by hemodialysis. Thus, hemodialysis certainly reduces plasma ascorbate concentration

237 in hemodialysis patients.

238 For a long time, there has been concern about the accumulation and deposition of oxalate with

239 increased intake of vitamin $\mathrm{C}$ because oxalate is a breakdown product of vitamin $\mathrm{C}$ and is

240 heavily excreted by the kidneys [29]. Oxalate crystallization occurs at levels above $30 \mathrm{mM}$ [30]

241 and high plasma oxalate levels were seen in hemodialysis patients [31-33]. However, in a recent

242 prospective case series exploring high-dose intravenous vitamin C (15-100 g) administration,

243 increased vitamin $\mathrm{C}$ intake was not associated with any cases of symptomatic renal stones and

244 kidney injury [34]. Moreover, significant side effects of vitamin C are not reported in any of

245 the mentioned controlled trials, including the most recent VITAMIN randomized trial [35].

246 CKD patients with higher levels of plasma calcium, phosphate, and parathyroid hormone have

247 a high risk of death because CKD often causes abnormal calcium and phosphate metabolism 
248 and hyperparathyroidism [36-40]. Therefore, it is important to control the plasma calcium,

249 phosphate, and parathyroid hormone levels in the non-hemodialysis CKD and hemodialysis

250 patients [41]. Through a systematic review and meta-analysis, Ke et al. [42] have reported that

251 vitamin $\mathrm{C}$ supplementation in CKD patients has no positive effect that influence the plasma

252 phosphate or parathyroid hormone levels, but it increase plasma calcium levels in the short term.

253 In the present study, we could not detect any correlation between plasma ascorbate and plasma

254 calcium, phosphate, and parathyroid hormone levels in Japanese hemodialysis patients.

255 Meanwhile, we only found the positive correlation between plasma ascorbate and plasma

256 potassium levels in Japanese hemodialysis patients. Perhaps Japanese hemodialysis patients

257 that have dietary potassium restrictions to prevent hyperkalemia may limit their consumption

258 of fresh vegetables and fruits that are rich in ascorbate. Thereby, there is a possibility that

259 Japanese hemodialysis patients have low plasma ascorbate levels.

260 Recently, the increase of frailty in the elderly has become a social problem globally. The

261 Dialysis Morbidity and Mortality Wave 2 cohort study revealed that $>60 \%$ of end-stage kidney

262 disease patients over the age of 40 met a definition of frailty, which impairs the prognosis [43].

263 Ascorbate is known to be one of the anti-aging factors because of its strong antioxidant 
264 properties [3]. Therefore, ascorbate may be causally associated with life prognosis and aging in

265 hemodialysis patients.

\section{Conclusion}

268 Japanese hemodialysis patients have low plasma ascorbate levels and are likely to develop

269 scurvy. Furthermore, their plasma ascorbate levels are reduced by approximately $40 \%$ by a

270 single hemodialysis. The cause of the low plasma ascorbate levels in hemodialysis patients may

271 be due to the decreased intake of ascorbate from fresh fruits and vegetables due to the strict

272 restriction of potassium intake. To avoid the development of scurvy in hemodialysis patients,

273 it is necessary to consume sufficient ascorbate from supplements or medicine because of the

274 body's inability to synthesize ascorbate.

275 
277 This study was supported by Japan Society for the Promotion of Science (JSPS) KAKENHI

278 Grant Number 19H04043 (Ishigami A).

279

280 Availability of data and materials

281 The datasets generated during the current study are available from the corresponding author on

282 reasonable request.

283

284 Authors' contributions

285 MI, TT, WY, NM, and AI designed the research; MI, TT, YT, AS, TY, and AI conducted the

286 experiments; YD, MI, TT, WY, and AI analyzed the data; and YD, MI, TT, YT, AS, TY, WY,

$287 \mathrm{NM}$, and AI wrote the manuscript and had primary responsibility for the final content of the

288 manuscript. All authors read and approved the final manuscript.

289

290 Disclosure statement

291 The authors declare no conflicts of interest. 


\section{References}

294

295 1. Linster CL, Van Schaftingen E: Vitamin C. Biosynthesis, recycling and degradation in mammals. FEBS J 2007, 274(1):1-22.

297

298

2. Winterbourn CC: Reconciling the chemistry and biology of reactive oxygen species.

299 Nature chemical biology 2008, 4(5):278-286.

302

3. Kondo Y, Sasaki T, Sato Y, Amano A, Aizawa S, Iwama M, Handa S, Shimada N, Fukuda M, Akita M et al: Vitamin C depletion increases superoxide generation in brains of SMP30/GNL knockout mice. Biochem Biophys Res Commun 2008, 377(1):291-296.

4. Lane DJ, Lawen A: Ascorbate and plasma membrane electron transport--enzymes vs

304 5. Mandl J, Szarka A, Banhegyi G: Vitamin C: update on physiology and pharmacology. efflux. Free Radic Biol Med 2009, 47(5):485-495.

6. Amano A, Tsunoda M, Aigaki T, Maruyama N, Ishigami A: Effect of ascorbic acid Br J Pharmacol 2009, 157(7):1097-1110. 
309 7. Furusawa H, Sato Y, Tanaka Y, Inai Y, Amano A, Iwama M, Kondo Y, Handa S, Murata A, Nishikimi $\mathrm{M}$ et al: Vitamin $\mathrm{C}$ is not essential for carnitine biosynthesis in vivo: verification in vitamin C-depleted senescence marker protein30/gluconolactonase knockout mice. Biol Pharm Bull 2008, 31(9):1673-1679.

313 8. Kondo Y, Inai Y, Sato Y, Handa S, Kubo S, Shimokado K, Goto S, Nishikimi M, Maruyama N, Ishigami A: Senescence marker protein 30 functions as gluconolactonase in L-ascorbic acid biosynthesis, and its knockout mice are prone to scurvy. Proc Natl Acad Sci U S A 2006, 103(15):5723-5728.

317 9. Nishikimi M, Koshizaka T, Ozawa T, Yagi K: Occurrence in humans and guinea pigs of the gene related to their missing enzyme L-gulono-gamma-lactone oxidase. Arch Biochem Biophys 1988, 267(2):842-846.

10. Panchal S, Schneider C, Malhotra K: Scurvy in a hemodialysis patient. Rare or ignored? Hemodial Int 2018, 22(S2):S83-S87.

322 11. Kimata N, Tsuchiya K, Akiba T, Nitta K: Differences in the Characteristics of Dialysis 40(4):275-279. 
325 12. Sullivan JF, Eisenstein AB: Ascorbic acid depletion during hemodialysis. JAMA 1972, 220(13):1697-1699.

327

13. Ponka A, Kuhlback B: Serum ascorbic acid in patients undergoing chronic hemodialysis. Acta Med Scand 1983, 213(4):305-307.

14. DeBari VA, Frank O, Baker H, Needle MA: Water soluble vitamins in granulocytes, erythrocytes, and plasma obtained from chronic hemodialysis patients. Am J Clin Nutr 1984, 39(3):410-415.

15. Papastephanidis C, Agroyannis B, Tzanatos-Exarchou H, Orthopoulos B, Koutsicos D, deficiency in hemodialysed patients. Int J Artif Organs 1987, 10(3):163-165. undergoing chronic haemodialysis. Eur J Clin Pharmacol 1999, 55(7):527-532. Descomps B, Canaud B: Convective and diffusive losses of vitamin C during haemodiafiltration session: a contributive factor to oxidative stress in haemodialysis patients. Nephrol Dial Transplant 2002, 17(3):422-427. 
341 18. Loughrey CM, Young IS, Lightbody JH, McMaster D, McNamee PT, Trimble ER: Oxidative stress in haemodialysis. QJM 1994, 87(11):679-683.

343 19. National Kidney F: K/DOQI clinical practice guidelines for chronic kidney disease: evaluation, classification, and stratification. Am J Kidney Dis 2002, 39(2 Suppl 1):S1266.

346 20. Kodama Y, Kishimoto Y, Muramatsu Y, Tatebe J, Yamamoto Y, Hirota N, Itoigawa Y, bronchial asthma. Clin Respir J 2017, 11(6):915-924. dehydroascorbic acid in mouse tissues and plasma by using tris(2acid/ethylenediaminetetraacetic acid solution. Biological \& pharmaceutical bulletin 2010, 33(3):364-369. filtration rate or on dialysis: a review. Semin Dial 2013, 26(5):546-567. 
357

23. Schleicher RL, Carroll MD, Ford ES, Lacher DA: Serum vitamin C and the prevalence of vitamin C deficiency in the United States: 2003-2004 National Health and Nutrition Examination Survey (NHANES). Am J Clin Nutr 2009, 90(5):1252-1263.

24. Young JI, Züchner S, Wang G: Regulation of the Epigenome by Vitamin C. Annu Rev Nutr 2015, 35:545-564.

25. Marik PE, Liggett A: Adding an orange to the banana bag: vitamin C deficiency is common in alcohol use disorders. Crit Care 2019, 23(1):165.

26. Honore PM, Spapen HD, Marik P, Boer W, Oudemans-van Straaten H: Dosing vitamin $\mathrm{C}$ in critically ill patients with special attention to renal replacement therapy: a narrative review. Ann Intensive Care 2020, 10(1):23.

27. Raimann JG, Abbas SR, Liu L, Larive B, Beck G, Kotanko P, Levin NW, Handelman G, Trial FHN: The effect of increased frequency of hemodialysis on vitamin C concentrations: an ancillary study of the randomized Frequent Hemodialysis Network (FHN) daily trial. BMC Nephrol 2019, 20(1):179.

28. Deicher R, Ziai F, Bieglmayer C, Schillinger M, Horl WH: Low total vitamin C plasma level is a risk factor for cardiovascular morbidity and mortality in hemodialysis patients. J Am Soc Nephrol 2005, 16(6):1811-1818. 
374 29. Elder TD, Wyngaarden JB: The biosynthesis and turnover of oxalate in normal and hyperoxaluric subjects. J Clin Invest 1960, 39:1337-1344.

30. Morgan SH, Purkiss P, Watts RW, Mansell MA: Oxalate dynamics in chronic renal failure. Comparison with normal subjects and patients with primary hyperoxaluria. Nephron 1987, 46(3):253-257.

31. Ogawa Y, Machida N, Ogawa T, Oda M, Hokama S, Chinen Y, Uchida A, Morozumi M, Sugaya K, Motoyoshi Y et al: Calcium oxalate saturation in dialysis patients with on plasma oxalate in patients on regular haemodialysis. Nephrol Dial Transplant 1991, $6(6): 440-443$. leads to plasma calcium oxalate supersaturation in hemodialysis patients. Am J Kidney Dis 2005, 45(3):540-549.

34. Prier M, Carr AC, Baillie N: No Reported Renal Stones with Intravenous Vitamin C Administration: A Prospective Case Series Study. Antioxidants (Basel) 2018, 7(5). 
With Septic Shock: The VITAMINS Randomized Clinical Trial. JAMA 2020.

36. Tentori F, Blayney MJ, Albert JM, Gillespie BW, Kerr PG, Bommer J, Young EW, levels of serum calcium, phosphorus, and PTH: the Dialysis Outcomes and Practice Patterns Study (DOPPS). Am J Kidney Dis 2008, 52(3):519-530.

37. Block GA, Klassen PS, Lazarus JM, Ofsthun N, Lowrie EG, Chertow GM: Mineral metabolism, mortality, and morbidity in maintenance hemodialysis. J Am Soc Nephrol 2004, 15(8):2208-2218. primarily and consistently controlled in prevalent hemodialysis patients. Ther Apher Dial 2013, 17(2):221-228. 
varying indicators of bone disease in maintenance hemodialysis patients. Kidney Int 2006, 70(4):771-780.

40. Floege J, Kim J, Ireland E, Chazot C, Drueke T, de Francisco A, Kronenberg F, Marcelli 2011, 26(6):1948-1955.

414

42. Ke G, Huang J, Zhu Y, Yang J, Zhang Y, Chen L, Hu J, Tao S, Hu Y, Yang D et al: Effect of Ascorbic Acid on Mineral and Bone Disorders in Hemodialysis Patients: a patients. J Am Soc Nephrol 2007, 18(11):2960-2967. 


\section{$424 \quad$ Figure Legends}

425

426 Fig. 1 Plasma ascorbate levels in non-hemodialysis CKD stage G3-G5 patients and pre- and

427 post-dialysis plasma ascorbate levels in CKD stage G5D hemodialysis patients. Total ascorbate

428 levels were determined as described in Methods. (a, b) Dots in boxplots are expressed ascorbate

429 levels in CKD stage G3-G5 $(n=27)$ and hemodialysis $(n=19)$ patients. Center lines are

430 expressed median value of each groups. (c) Distributions of ascorbate levels in each group. $P$

$431<0.05$ by (a) Welch's t-test and (b) paired t-test.

432

433 Fig. 2 Scatterplots between clinical characteristics and pre-dialysis plasma ascorbate levels in

434 hemodialysis patients. Dots are expressed data of individual hemodialysis patients $(\mathrm{n}=19)$. Blue

435 and red lines are regression lines of data sets between ascorbate levels and clinical data. Pearson

436 correlation coefficients are described in Table 2.

437

438 Fig. 3 Plasma ascorbate levels in healthy controls $(n=28)$ [20] and chronic obstructive

439 pulmonary disease (COPD) $(n=39)$ [20], non-hemodialysis chronic kidney disease (CKD) stage

440 G3-G5 ( $\mathrm{n}=27)$, and hemodialysis $(\mathrm{n}=19)$ patients. Ascorbate (blue column) and DHA (yellow 
441 column) levels were determined as described in Methods. The average concentration of

442 ascorbate in the plasma of healthy humans is $40-60 \mu \mathrm{M}$ (blue zone). There is a risk of

443 developing scurvy when the plasma ascorbate concentration drops to below $11 \mu \mathrm{M}$ (red zone).

444 Values are expressed as a mean \pm SEM. $P<0.05$ by one-way ANOVA followed Tukey-Kramer

445 test.

446 
Table 1 Clinical characteristics of CKD stage G3-G5 and hemodialysis patients

\begin{tabular}{|c|c|c|c|}
\hline Characteristic & Normal range & $\begin{array}{l}\text { CKD stage G3-G5 } \\
(n=27)\end{array}$ & $\begin{array}{l}\text { Hemodialysis } \\
(n=19)\end{array}$ \\
\hline Age (years) & & $83.9 \pm 1.3$ & $78.9 \pm 2.5$ \\
\hline Sex (male/female) & & $10 / 17$ & $9 / 10$ \\
\hline \multirow[t]{2}{*}{ White blood cell $\left(\mathrm{x} 10^{3} / \mu \mathrm{l}\right)$} & M: 3.9-9.8 & M: $6.7 \pm 0.6$ & $M: 6.5 \pm 0.4$ \\
\hline & F: $3.5-9.1$ & $F: 7.0 \pm 0.4$ & F: $7.1 \pm 1.1$ \\
\hline \multirow[t]{2}{*}{ Hemoglobin (g/dL) } & M: $13.5-17.6$ & $\mathrm{M}: 12.1 \pm 0.4$ & $\mathrm{M}: 11.3 \pm 0.2$ \\
\hline & F: $11.3-15.2$ & F: $11.8 \pm 0.3$ & F: $11.2 \pm 0.2$ \\
\hline \multirow[t]{2}{*}{ Hematocrit (\%) } & M: $39.8-51.8$ & M: $36.5 \pm 1.2$ & M: $34.3 \pm 0.8$ \\
\hline & F: $33.4-44.9$ & $F: 36.2 \pm 0.7$ & F: $35.1 \pm 0.8$ \\
\hline \multirow[t]{2}{*}{ Platelet $\left(\mathrm{x} 10^{4} / \mu \mathrm{l}\right)$} & M: $13.1-36.2 *$ & M: $20.5 \pm 2.0$ & M: $23.8 \pm 2.3$ \\
\hline & F: $13.0-36.9$ & $F: 22.1 \pm 1.4$ & F: $16.0 \pm 1.9$ \\
\hline Total protein $(\mathrm{g} / \mathrm{dL})$ & $6.7-8.3 *$ & $6.9 \pm 0.1$ & $6.3 \pm 0.1$ \\
\hline Albumin (g/dL) & $3.8-5.2 *$ & $3.6 \pm 0.1$ & $3.1 \pm 0.1$ \\
\hline C-Reactive protein (mg/dL) & $<0.3$ & $0.4 \pm 0.1$ & $0.8 \pm 0.6$ \\
\hline AST (IU/L) & $37.0-125.0$ & $21.2 \pm 1.0$ & $24.7 \pm 8.4$ \\
\hline Blood urea nitrogen $(\mathrm{mg} / \mathrm{dL})$ & $8.0-22.0 *$ & $32.9 \pm 2.8$ & $62.7 \pm 3.1$ \\
\hline \multirow[t]{2}{*}{ Creatinine (mg/dL) } & M: $0.6-1.0 *$ & M: $2.0 \pm 0.3$ & M: $10.4 \pm 0.6$ \\
\hline & $\mathrm{F}: 0.5-0.8 *$ & $\mathrm{~F}: 2.1 \pm 0.3$ & F: $7.4 \pm 0.4$ \\
\hline \multirow[t]{2}{*}{ Uric acid (mg/dL) } & M: 3.7-7.0 & M: $6.0 \pm 0.3$ & $\mathrm{M}: 5.9 \pm 0.5$ \\
\hline & F: $2.5-7.0$ & $F: 6.3 \pm 0.4$ & $\mathrm{~F}: 5.9 \pm 0.5$ \\
\hline Sodium (mEq/L) & $136.0-147.0 *$ & $140.4 \pm 0.7$ & $137.7 \pm 0.8$ \\
\hline Potassium (mEq/L) & $3.6-5.0 *$ & $4.4 \pm 0.1$ & $4.9 \pm 0.2$ \\
\hline Calcium (mg/dL) & $8.5-10.2 *$ & $9.1 \pm 0.1$ & $8.5 \pm 0.1$ \\
\hline Phosphorus (mg/dL) & $2.4-4.3 *$ & $3.8 \pm 0.1$ & $5.1 \pm 0.3$ \\
\hline Triglyceride (mg/dL) & $50.0-149.0 *$ & $150.9 \pm 14.1$ & $108.7 \pm 7.5$ \\
\hline Total cholesterol (mg/dL) & $150.0-219.0 *$ & $200.9 \pm 11.3$ & $152.8 \pm 8.7$ \\
\hline LDL cholesterol (mg/dL) & $70.0-139.0 *$ & $109.5 \pm 6.8$ & $81.2 \pm 6.1$ \\
\hline \multirow[t]{2}{*}{ Iron $(\mu \mathrm{g} / \mathrm{dL})$} & M: $54.0-200.0 *$ & M: $66.2 \pm 4.4$ & M: $62.8 \pm 14.9$ \\
\hline & F: $48.0-154.0$ & F: $76.6 \pm 6.8$ & $F: 47.5 \pm 15.5$ \\
\hline \multirow[t]{2}{*}{ TIBC $(\mu \mathrm{g} / \mathrm{dL})$} & M: 253.0-365.0 & M: $234.6 \pm 5.2$ & M: $251.7 \pm 12.8$ \\
\hline & $\mathrm{F}: 246.0-410.0$ & $F: 253.6 \pm 8.6$ & $F: 230.9 \pm 15.5$ \\
\hline Ferritin (ng/dL) & M: $3940-34,000$ & M: $159.6 \pm 27.3$ & M:105.9 \pm 27.9 \\
\hline
\end{tabular}




\begin{tabular}{ll:ll} 
& F: $360-11,400$ & F: $177.2 \pm 60.7$ & F: $117.3 \pm 25.1$ \\
B2-Microglobulin (mg/L) & $1.0-1.9^{*}$ & $5.1 \pm 0.6$ & $27.0 \pm 1.7$ \\
Prealbumin (mg/dL) & $22.0-40.0$ & $23.2 \pm 1.1$ & $24.1 \pm 1.3$ \\
HbA1c (\%) & $4.6-6.2$ & $6.1 \pm 0.1$ & $5.8 \pm 0.2$ \\
\hline
\end{tabular}

Values are presented as the mean \pm SEM. * significant difference at $p<0.05$

M, male; F, female; CKD, chronic kidney disease; AST, aspartate aminotransferase; LDL, lowdensity lipoprotein; TIBC, total iron-binding capacity; HbA1, hemoglobin A1c 
Table 2 Pearson correlation coefficients (r) between clinical characteristics and pre-dialysis plasma ascorbate levels in hemodialysis patients

\begin{tabular}{|c|c|c|}
\hline Characteristic & $\mathrm{r}$ & $p$-value \\
\hline Age & 0.44 & 0.056 \\
\hline Dry weight & 0.02 & 0.934 \\
\hline White blood cell & 0.12 & 0.617 \\
\hline Hemoglobin & 0.18 & 0.461 \\
\hline Hematocrit & 0.22 & 0.362 \\
\hline Platelet & -0.38 & 0.104 \\
\hline Total protein & 0.02 & 0.944 \\
\hline Albumin & -0.12 & 0.632 \\
\hline C-Reactive protein & 0.40 & 0.087 \\
\hline AST & 0.30 & 0.206 \\
\hline Blood urea nitrogen & 0.05 & 0.835 \\
\hline Creatinine & -0.12 & 0.633 \\
\hline Uric acid & -0.05 & 0.828 \\
\hline Sodium & 0.15 & 0.537 \\
\hline Potassium * & 0.60 & 0.006 \\
\hline Calcium & 0.05 & 0.829 \\
\hline Phosphorus & -0.14 & 0.578 \\
\hline Triglyceride & 0.30 & 0.206 \\
\hline Total cholesterol & 0.32 & 0.179 \\
\hline LDL cholesterol & 0.18 & 0.454 \\
\hline Iron & 0.01 & 0.962 \\
\hline TIBC & -0.33 & 0.161 \\
\hline Ferritin & 0.44 & 0.060 \\
\hline$\beta 2$-Microglobulin & 0.18 & 0.465 \\
\hline Prealbumin & 0.00 & 0.995 \\
\hline $\mathrm{HbAlc}$ & -0.19 & 0.430 \\
\hline Parathyroid hormone & -0.06 & 0.816 \\
\hline
\end{tabular}

$* p$-value $<0.05$

AST, aspartate aminotransferase; LDL, low-density lipoprotein; TIBC, total iron-binding capacity; $\mathrm{HbA1}$, hemoglobin A1c 
a

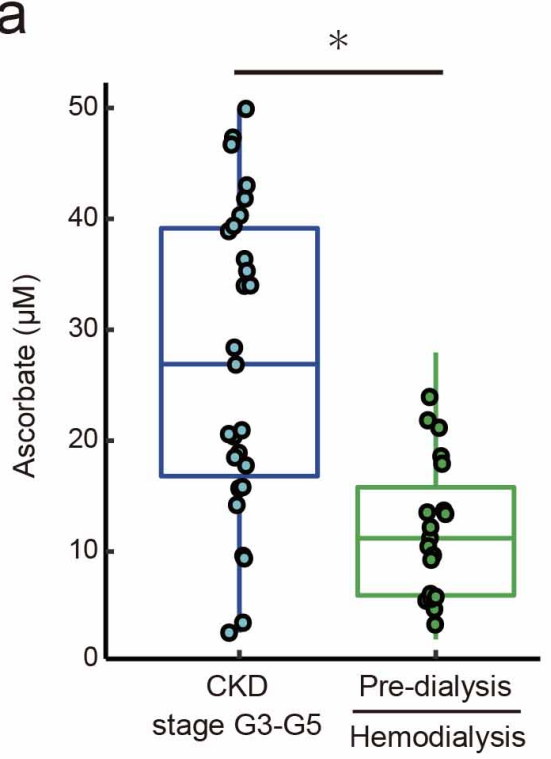

b

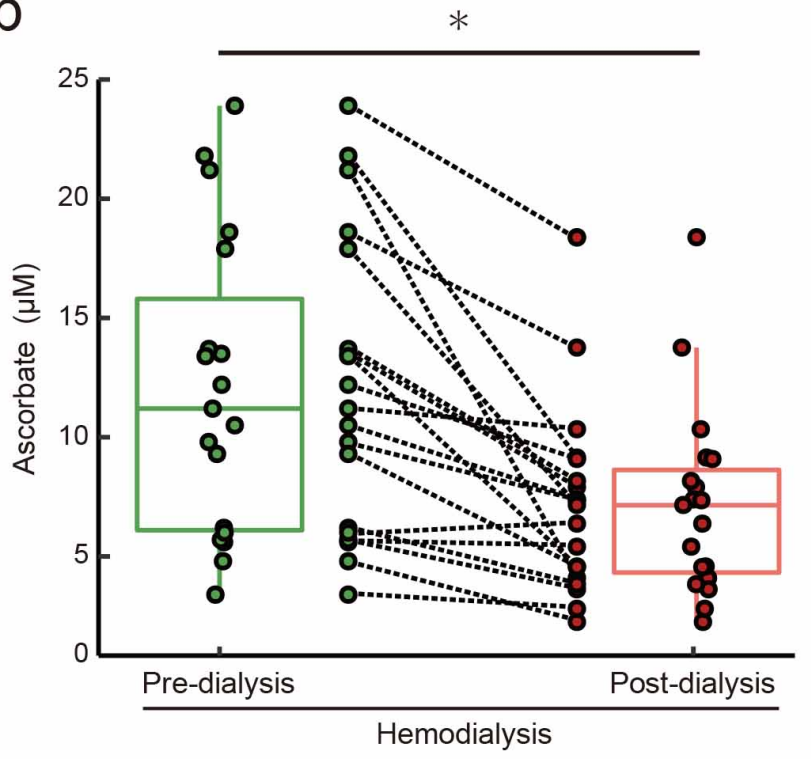

C

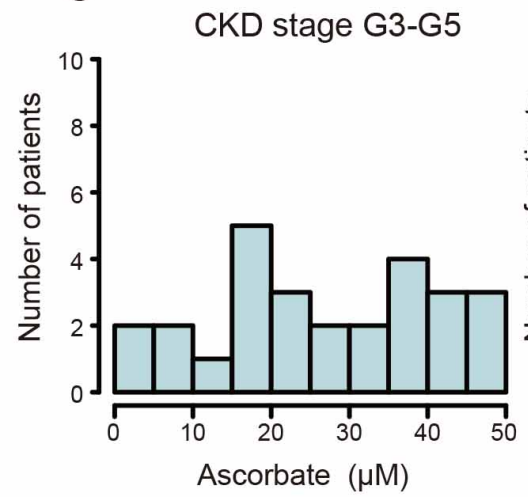

Post-dialysis

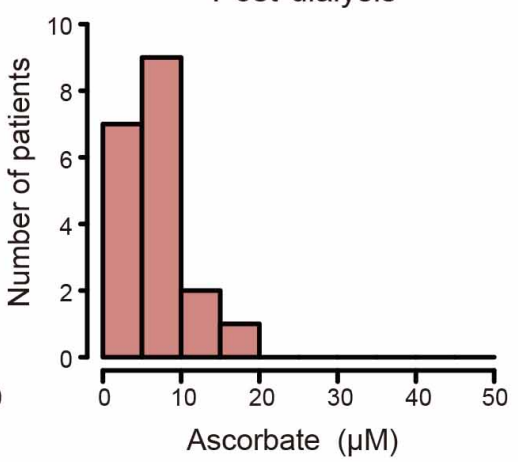

Figure 1 

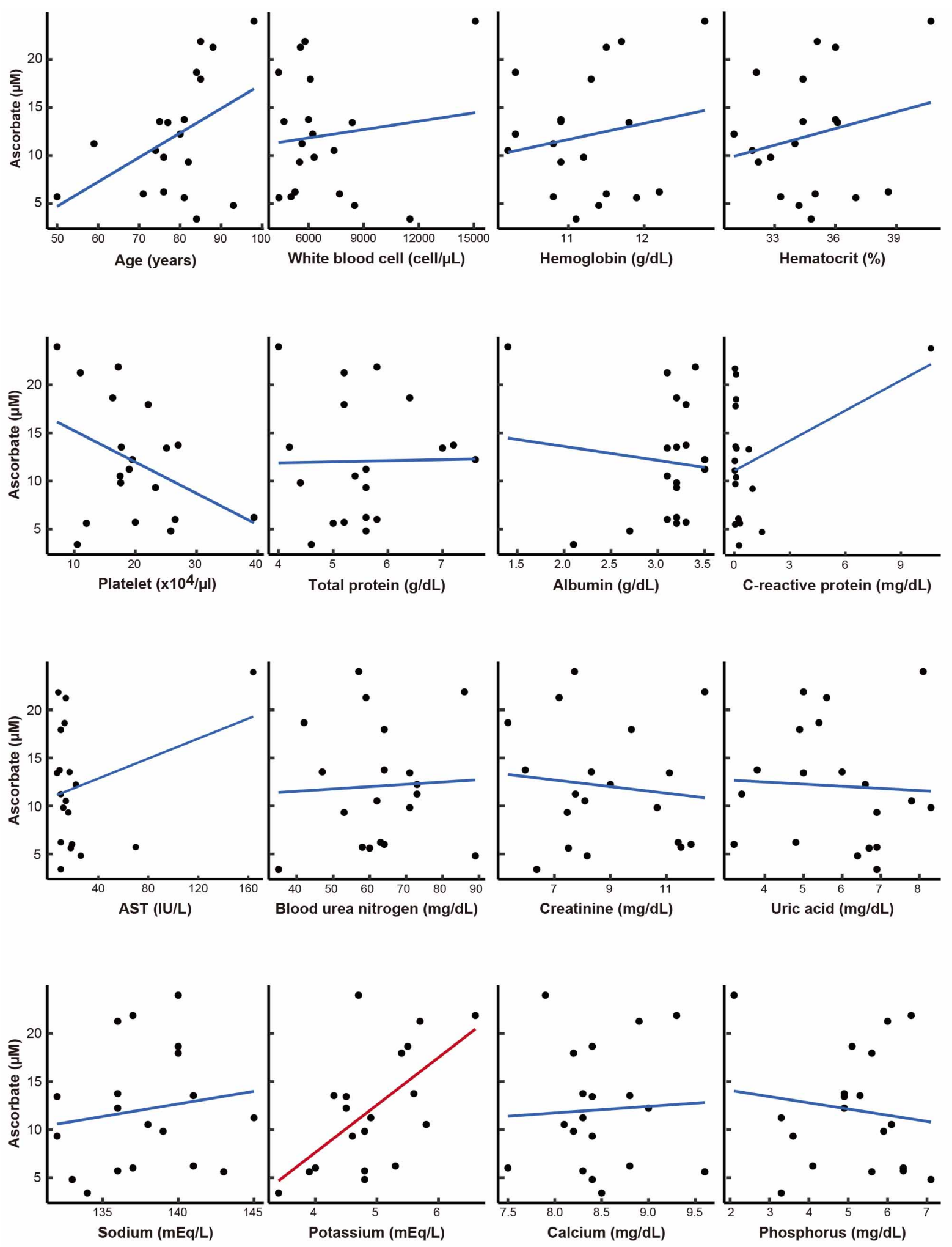

Figure 2 
451
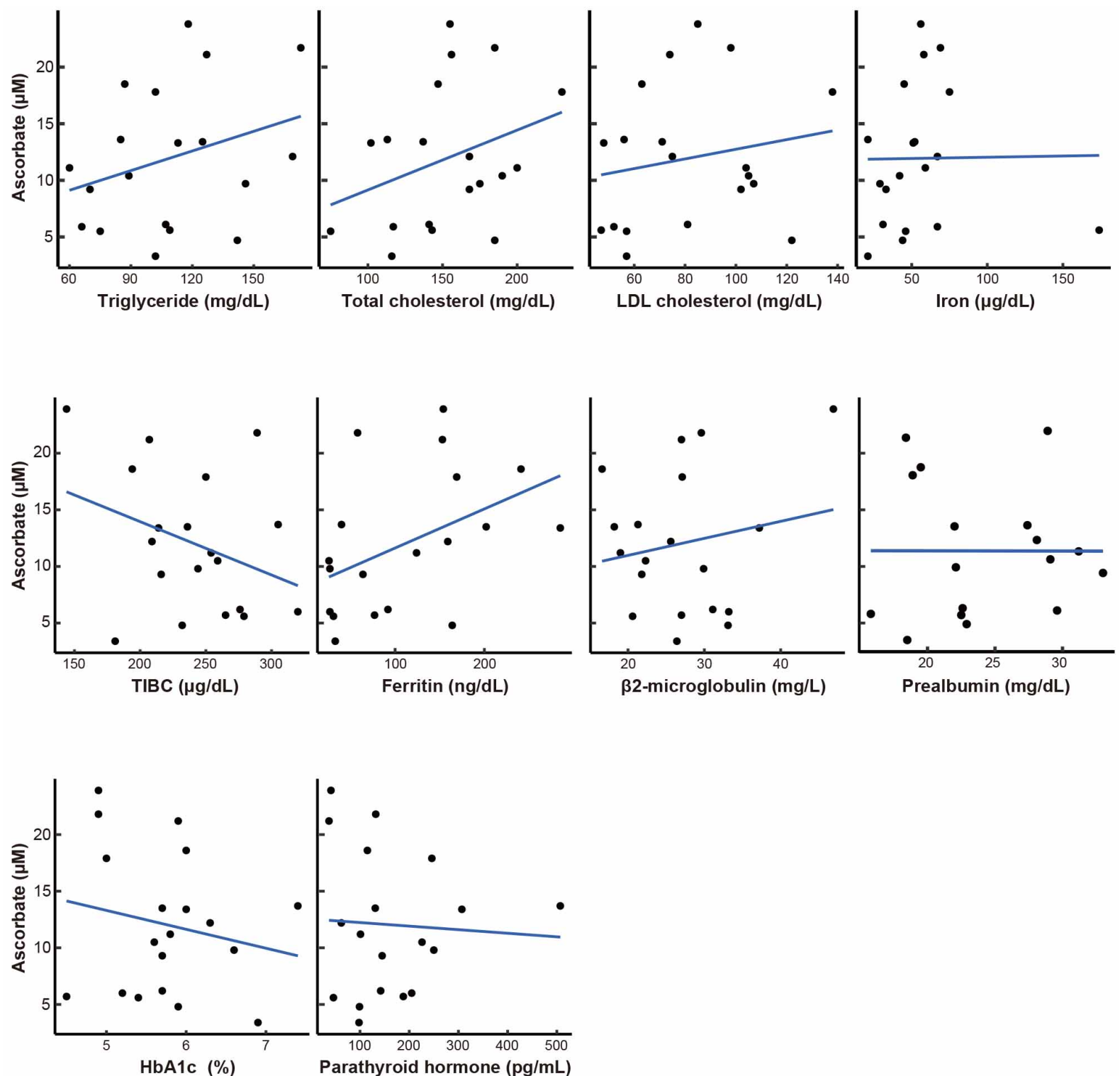

Figure 2 
453

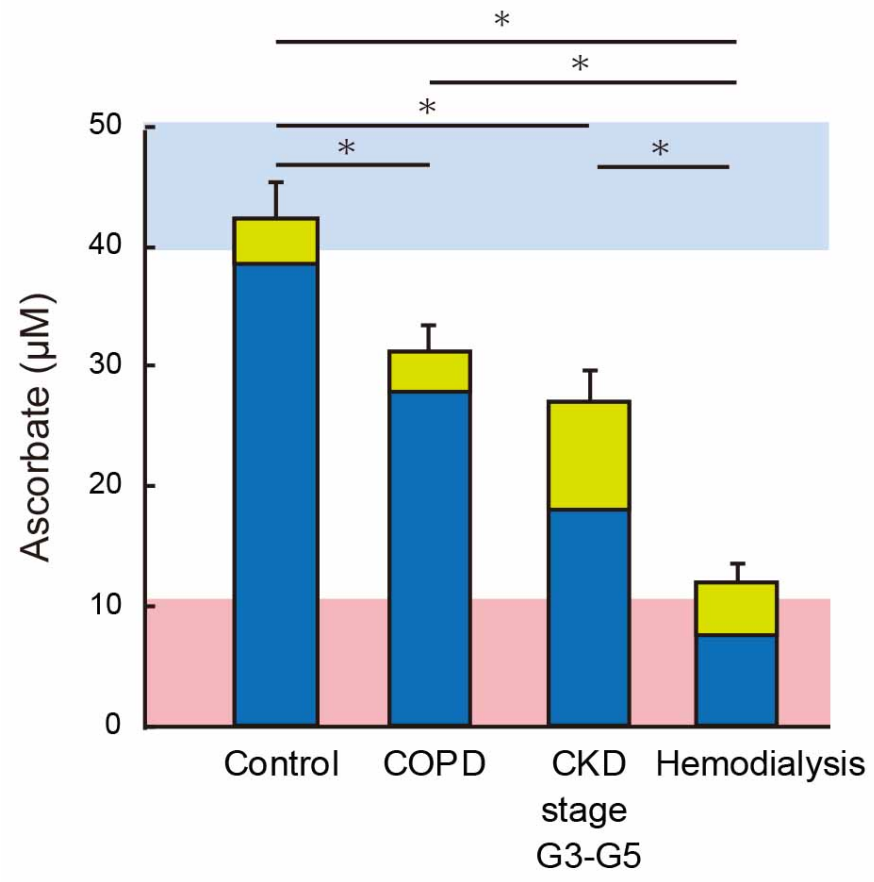

Figure 3 
a

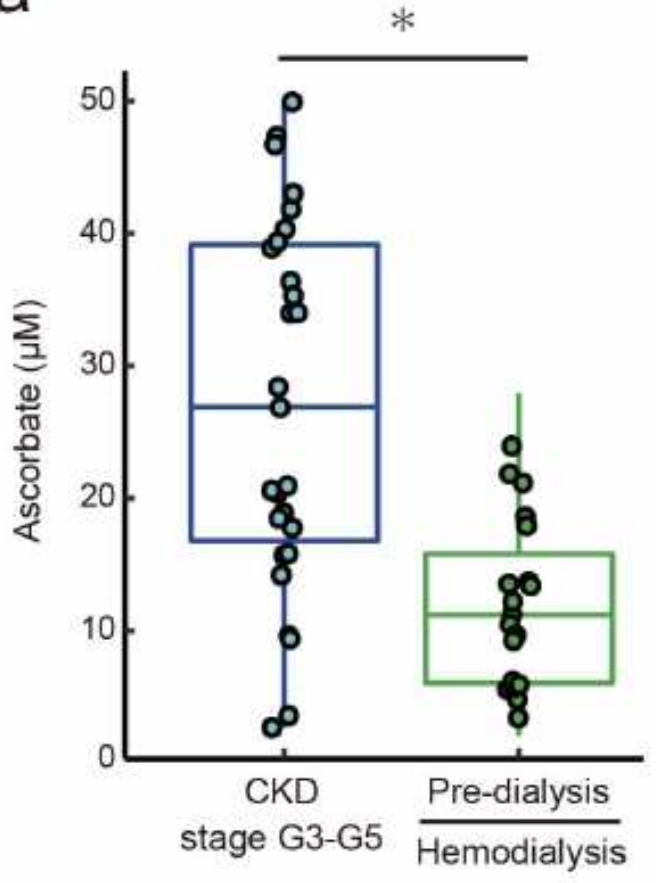

b

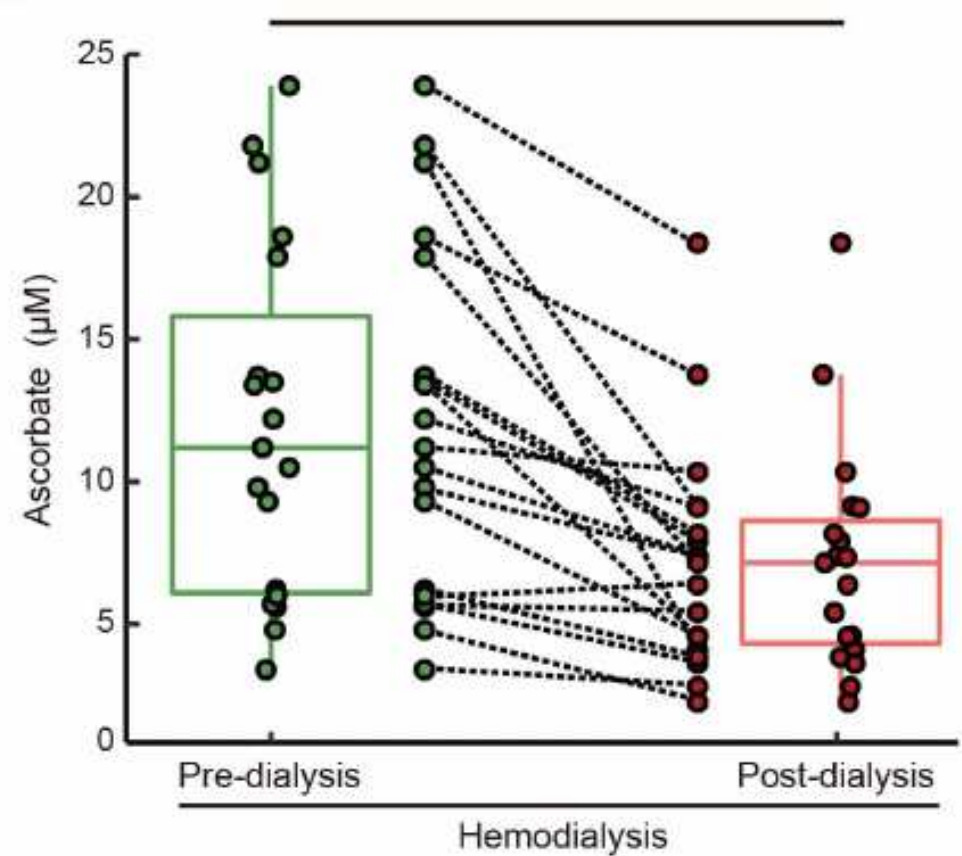

Pre-dialysis

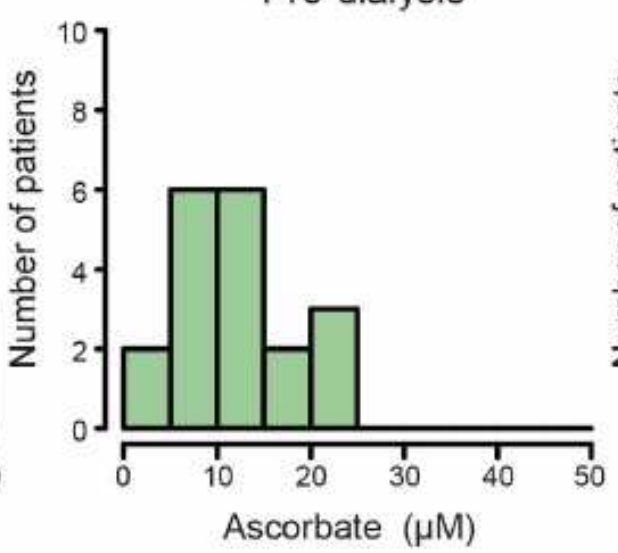

Post-dialysis

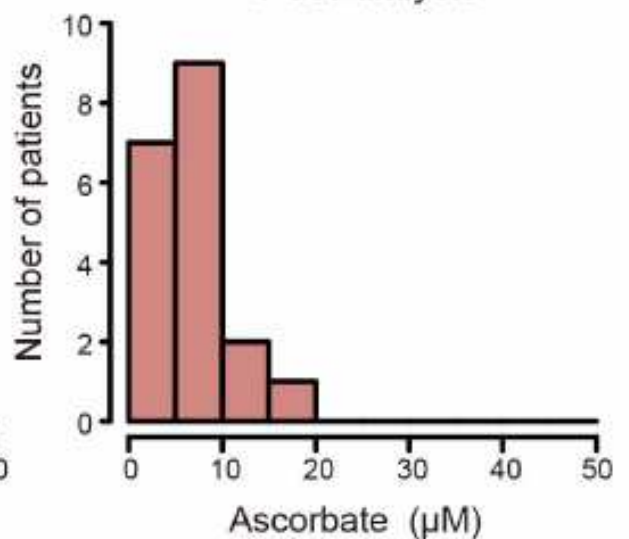

Figure 1

Plasma ascorbate levels in non-hemodialysis CKD stage G3-G5 patients and pre- and post-dialysis plasma ascorbate levels in CKD stage G5D hemodialysis patients. Total ascorbate levels were determined as described in Methods. $(a, b)$ Dots in boxplots are expressed ascorbate levels in CKD stage G3-G5 $(n=27)$ and hemodialysis $(n=19)$ patients. Center lines are expressed median value of each groups. (c) Distributions of ascorbate levels in each group. $\mathrm{P}<0.05$ by (a) Welch's t-test and (b) paired t-test. 

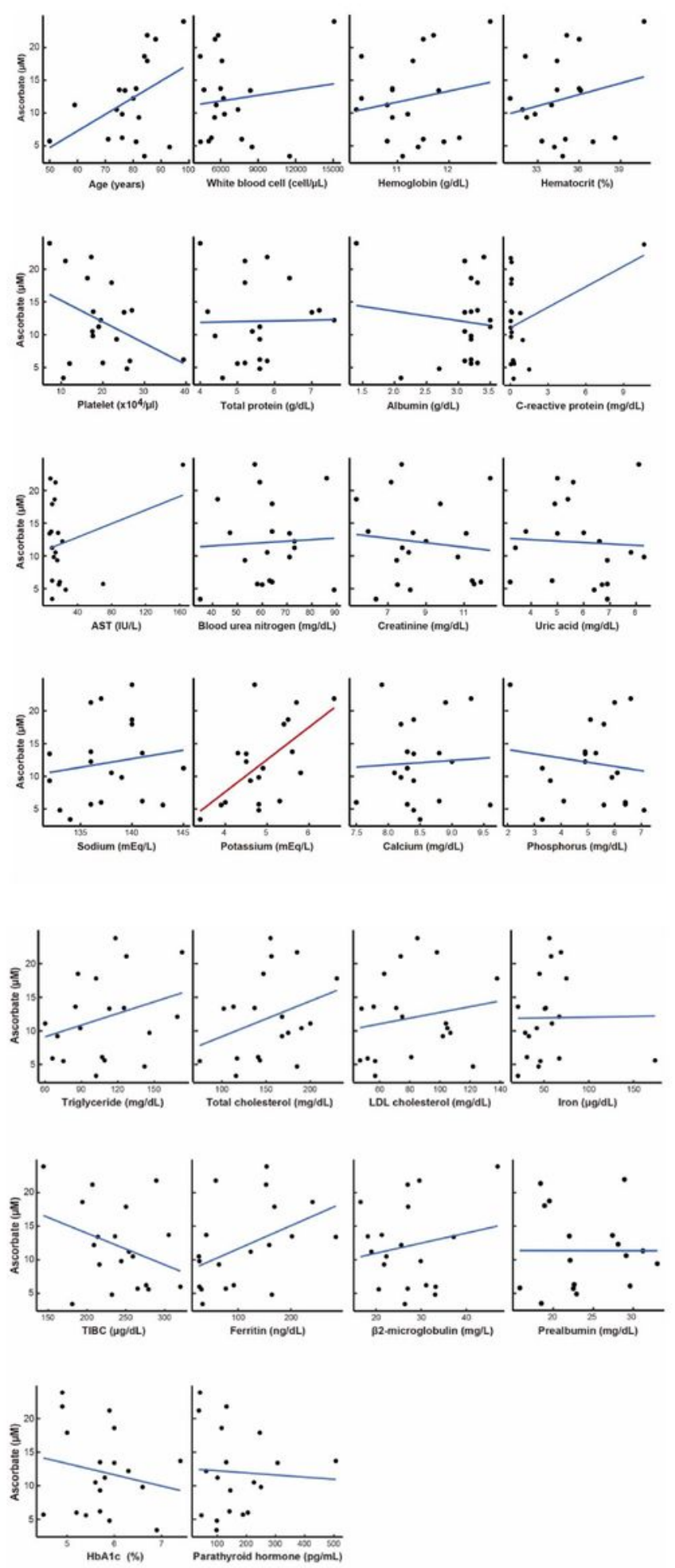

Figure 2

Scatterplots between clinical characteristics and pre-dialysis plasma ascorbate levels in hemodialysis patients. Dots are expressed data of individual hemodialysis patients $(n=19)$. Blue and red lines are regression lines of data sets between ascorbate levels and clinical data. Pearson correlation coefficients are described in Table 2. 


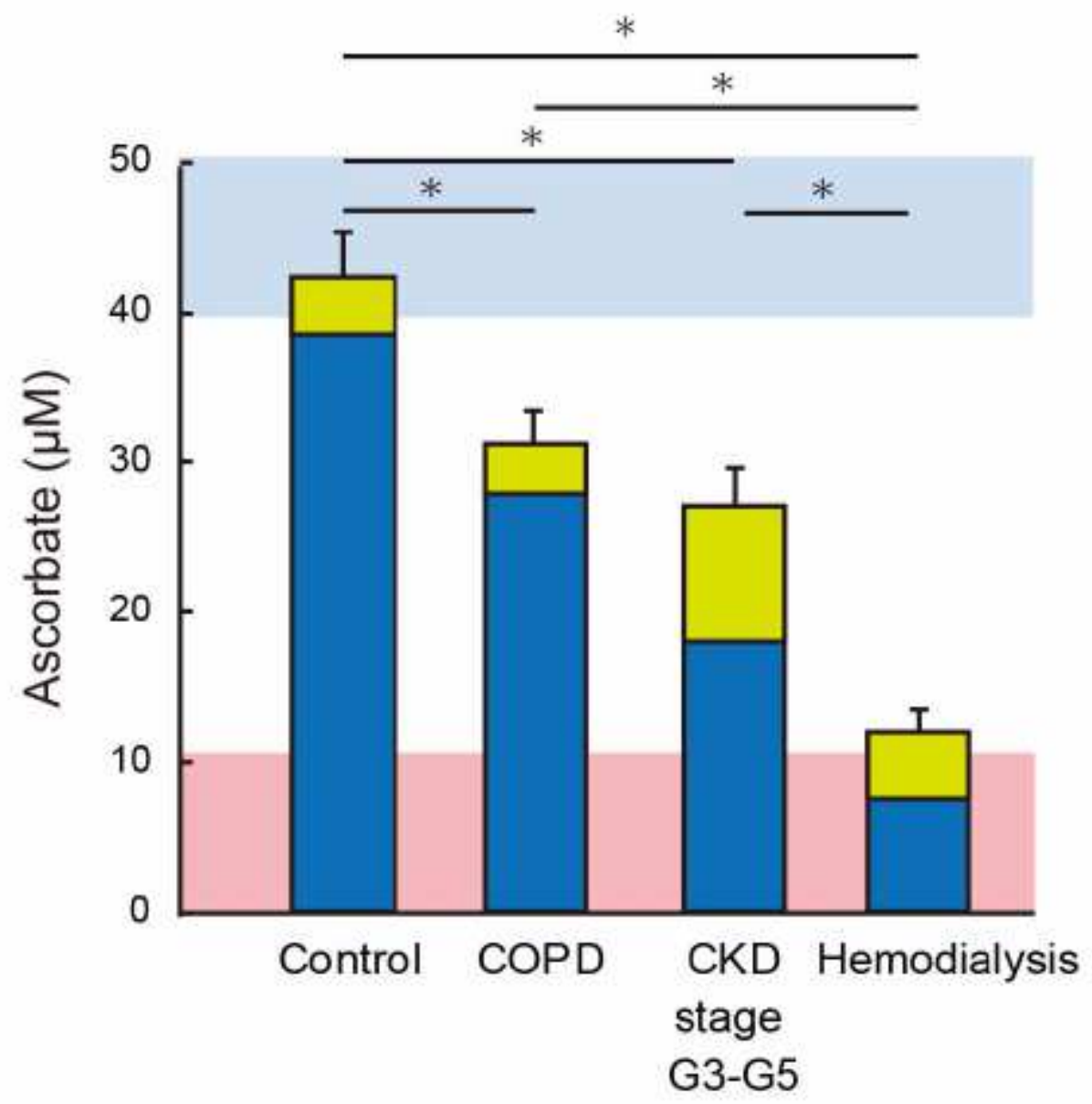

Figure 3

Plasma ascorbate levels in healthy controls $(n=28)[20]$ and chronic obstructive pulmonary disease (COPD) ( $n=39)$ [20], non-hemodialysis chronic kidney disease (CKD) stage G3-G5 ( $n=27)$, and hemodialysis ( $n=19$ ) patients. Ascorbate (blue column) and DHA (yellow column) levels were determined as described in Methods. The average concentration of ascorbate in the plasma of healthy humans is 40-60 $\mu \mathrm{M}$ (blue zone). There is a risk of developing scurvy when the plasma ascorbate concentration drops to below $11 \mu \mathrm{M}$ (red zone). Values are expressed as a mean \pm SEM. $\mathrm{P}<0.05$ by one-way ANOVA followed Tukey-Kramer test. 